\title{
TROCAS DE MASSA E DE ENERGIA ENTRE SUPERFÍCIES NATURAIS E A ÄTMOSFERA.
}

\author{
Arí de O. MARQUES FILHO'
}

\begin{abstract}
RESUMO - A modelização matemática das trocas de massa e de energia resultantes da interação entre vegetação e atmosfera é tratada pela combinação de análises das equações diferenciais representando a conservação de propriedades do ar (temperatura e umidade) com a equação do balanço de energia local do meio vegetal. Fluxos e fontes de calor e de massa, perfis de temperatura e umidade foram calculados através das relaçōes matriciais das equaçōes diferenciais discretizadas. As transferências radiativas são defïnidas no modelo, considerando a vegetação como um meio túrbido, onde as superfícies vegetais são distribuídas de forma aleatória no espaço. Os resultados produzidos pelo modelo para floresta são comparados com medidas micrometeorológicas realizadas na Reserva Florestal Ducke, Manaus - AM.
\end{abstract}

Palavras Chaves: micrometeorologia, modelização matemática, evaporação, floresta.

Mass and Energy Exchange Betwenn Natural Sungaces and the Atmosphere.

\begin{abstract}
The mathematical modeling of encrgy and mass exchange in the vegetationatmosphere interaction is treated by combining the analysis of the differential equations representing air properties conservation (temperature, humidity) and local energy budget equation of vegetative medium. Fluxes, sources, temperature and humidity profiles were calculated by the matrix form of discrete differential equations. Radiative transfers are defined in the model, considering the vegetation as a turbid medium, where the vegetal surfaces are distributed randomly in the space. The results produced by the model for a forest are compared with micrometeorological measurements obtained at Reserva Florestal Ducke, Manaus - AM.
\end{abstract}

Keywords: Micrometeorology, mathematical modeling, evaporation, forest.

\section{INTRODUÇÃO}

Um modelo matemático que descreve os fluxos de massa e de energia das superfícies naturais para a atmosfera é apresentado neste estudo, onde os seguintes processos físicos e suas mutuais dependências são explorados: (i) radiação solar, a principal fonte de energia para o sistema solo-plantaatmosfera, determinando primariamente a distribuição de energia no interior da vegetação; (ii) radiação de onda longa das superfícies vegetais e do solo, que constituem uma contribuição interna para o balanço de energia do sistema; (iii) características turbulentas do movimento do ar acima e no interior da vegetação, focalizando especialmente os vórtices turbulentos de diferentes tamanhos, que devem atuar no transporte e distribuição dos fluxos locais para as outras zonas da vegetação ou da atmosfera. Esses movimentos tem um importante papel na definição dos perfis de temperatura e de umidade, determinam as variações no armazenamento de energia no interior da cobertura vegetal, e contribuem na composição dos fluxos globais do

\footnotetext{
Instituto Nacional de Pesquisas da Amazônia, Caixa Postal n 478, CEP 69083.000 Manaus Brasil.
} 
conjunto vegetativo para a atmosfera.

Uma primeira proposição para estudar as transferências de massa e de energia, envolvendo o balanço de energia das superfícies e usando equações diferenciais para descrever cada processo, foi feita por PHILIP (1964). A sua análise esteve restrita ao caso de regime permanente de escoamento e as fontes locais de calor e de massa no interior da vegetação foram parametrizadas ou descritas como dependentes das difusividades turbulentas locais, moduladas por um coeficiente que incorporaria alguns aspectos do conjunto vegetal, tais como, os espaçamentos típicos dos elementos foliares, dimensão característica desses elementos, espaço livre, etc. Nesse mesmo contexto, COWAN (1968) desenvolveu uma análise teórica, resultando em expressões analíticas explicitas para descrever as difusividades turbulentas no espaço ocupado pela vegetação. Nesse trabalho, as equações diferenciais que exprimem as transferências de massa e de calor foram também enfocadas, e as soluções apropriadas ao caso simples de distribuição foliar constante na vertical foram obtidas. PERRIER $(1967,1976)$ introduziu uma equação diferencial para a descrição da quantidade de movimento acima e no interior de coberturas vegetais, na qual as difusividades turbulentas são associadas à configuração estrutural da vegetação por meio de uma abordagem probabilística da distribuição espacial dos elementos vegetais. Análises diferenciais foram também aplicadas para explicar as variações da entalpia e do deficit de saturação, e concentrações de $\mathrm{CO}_{2}$ no ar, incluindo conceitos teóricos ou numéricos no desenvolvimento de soluções. BRUTSAERT (1979) apresentou um exemplo de solução analítica para o tipo de equações diferenciais referidas aqui. Seu tratamento é aplicável em condições de escoamento permanente sobre vegetação densa, onde as concentrações das propriedades do ar (temperatura, vapor d'água, etc.) ao nível dos elementos vegetais possam ser consideradas constantes.

Paralelamente, WAGGONER \& REIFSNYDER (1968), usando os conceitos de resistência aerodinâmica, resistência de camada limite e estomática, abrem uma linha de abordagem relativamente diferente na forma, mas essencialmente compatível nos seus aspectos substanciais com as idéias que dão suporte à descrição matemática das transferências nos termos já discutidos acima. O trabalho original desses autores compreendia a simulação dos perfis de temperatura e umidade do ar, e a descrição dos perfis de evaporação no interior da vegetação. WAGGONER et al. (1969) completaram a análise anterior, incluindo um tratamento para os fluxos de calor sensível. A formulação desse modelo em forma matricial foi feita por FURNIVAL et al. (1975), CHEN (1984), TUZET (1989), HALDDYN \& LINDROTH (1986), entre outros.

Recentemente, a definição de difusividades turbulentas ( $\mathrm{e}$ as trocas de massa/energia), seguindo a teoria de 
difusão e o seu corolário de proporcionalidade entre fluxos e gradientes, foram retomadas em alguns trabalhos, tais como, BACHE (1986b), MASSMAN (1987a,b), MASSMAN \& VAN DUKEN (1989), VAN DE GRIEND \& VAN BOXEL (1989) and SELLERS et al. (1989).

No presente estudo, tratamentos para o caso de regime não permanente de escoamento e para os termos de radiação de onda longa das superfícies vegetais e do solo são conjugados, e formam um modelo global para descrever as fontes, os perfis e os fluxos de massa (vapor d'água) e de energia, resultantes das interações entre as coberturas vegetais e a atmosfera.

\section{DESENVOLVIMENTO DO MODELO}

Análises sobre as transferências de vapor d'água e de calor acima e no interior da vegetação e sua conexão com o balanço de energia de cada camada são desenvolvidas numa abordagem unidimensional (eixo vertical) de regime não permanente sem qualquer contribuição significante de transportes advectivos. Admite-se também que as difusividades turbulentas associadas aos transportes de massa e de energia possam ser determinadas de forma independente como funções de $\mathbf{z}$ (coordenada vertical), seja a partir de uma hipótese sobre a sua variação no interior do corpo vegetativo, seja a partir do suporte de medidas, ou ainda pelo uso de funções preconizados por modelos independentes.

Nessas condições, o fenômeno das transfêrencias pode ser representado por três equações diferenciais, expressando em cada camada a conservação de calor (associada com o perfil de temperatura do ar), de massa (perfil de umidade específica do ar ) e o balanço de energia,

$$
\rho c_{p} \frac{\partial \theta(z, t)}{\partial t}+\frac{\partial}{\partial z}\left[-K_{\theta} \rho c_{p} \frac{\partial \theta(z, t)}{\partial z}\right]=S^{*}(z, t)
$$

$$
L_{v} \frac{\partial C(z, t)}{\partial t}+\frac{\partial}{\partial z}\left[-K_{v} L_{v} \frac{\partial C(z, t)}{\partial z}\right]=V^{*}(z, t)
$$

$$
\rho_{v g} C_{v g} \frac{\partial T_{s}(z, t)}{\partial t}+S^{*}(z, t)+V^{*}(z, t)=\frac{\partial}{\partial z}\left[F_{n c}(z, t)-G(t)\right]+\frac{\partial}{\partial z}\left[F_{1}(z, t)\right.
$$

Os termos do lado esquerdo das equaçōes 1 e 2 relacionados às derivadas verticais representam as variações dos fluxos acumulados de calor sensível e de calor latente, $\partial \mathrm{H}_{\mathrm{s}} / \partial \mathrm{z}$ e $\partial\left(\mathrm{L}_{\mathrm{v}} \mathrm{E}_{\mathrm{v}}\right) / \partial \mathrm{z}$, respectivamente. De outro lado, as 
parametrizações para as fontes de calor e de vapor d'água, $S^{*}$ e $V^{*}$, são feitas pelas equações:

$S^{*}(z, t)=\rho c_{p} \frac{\Theta_{s}(z, t)-\Theta(z, t)}{r_{b}} a(z)$

$$
V^{*}(z, t)=L_{v} \frac{C_{s}(z, t)-C(z, t)}{r_{b}+r_{s}} a(z)
$$

As condições iniciais e as condições nos limites do sistema físico impostas às equações 1 e 2 podem ser expressas pelos seguintes perfis das diferentes propriedades:

$$
\begin{array}{llll}
\mathrm{z}=0 & \Theta=\Theta(0, \mathrm{t}) & \mathrm{C}=\mathrm{C} \quad(0, \mathrm{t}) \\
\mathrm{z}=\mathrm{zr} & \Theta=\Theta(\mathrm{zr}, \mathrm{t}) & \mathrm{C}=\mathrm{C} \quad(\mathrm{zr}, \mathrm{t}) \\
\mathrm{t}=0 & \Theta=\Theta(\mathrm{z}, 0) & \mathrm{C}=\mathrm{C} \quad(\mathrm{z}, 0)
\end{array}
$$

\section{Abordagem matricial e solução númerica do modelo}

O processo adotado para transformar essas equações contínuas em formas discretas equivalentes baseia-se na definição das diferenças finitas:

$$
\begin{aligned}
& \Delta \theta_{z}=\theta_{i}-\theta_{i-1} \\
& \Delta^{2} \Theta_{z}=\Theta_{i+1}-\theta_{i}+\theta_{i-1} \\
& \Delta \theta_{t}=\theta_{t}-\Theta_{t-\Delta t} \\
& \Delta K_{z}=K_{i}-K_{i-1}
\end{aligned}
$$

Aplicando essas diferenças em cada camada e efetuando a soma das expressões individuais assim obtidas, da primeira camada (próxima da superfície do solo) até uma camada i qualquer, um termo geral pode ser evidenciado, que representa uma equação transformada válida para a camada i,

$$
\sum_{j=1}^{i} \rho c_{p} \frac{\Theta_{j(t)}-\Theta_{j(t-\Delta)}}{\Delta t} \Delta z-K_{i} \rho c_{p} \frac{\Theta_{i+1}-\Theta_{i}}{\Delta z}=\sum_{j=0}^{i} S_{j}
$$

onde, $\mathrm{S}$ é simplesmente $\mathrm{S}^{*} . \Delta \mathrm{z}$. É necessário ainda que esta última equação seja transformada para evidenciar uma relação direta entre as fontes de calor (S) e as temperaturas dos elementos vegetais $\left(\Theta_{\mathrm{s}}\right)$. Então, uma combinação das equações 4 e 6 fornece a equação modificada,

$\sum_{j=1}^{i} \rho c_{p} \frac{\Theta_{j(t)}-\Theta_{j(t-\Delta)}}{\Delta t} \Delta z-K_{i} \rho c_{p} \frac{\Theta_{i+1}-\Theta_{i}}{\Delta z}=\sum_{j=0}^{i} S_{j}+\frac{K_{i}}{\Delta z}\left(S_{i} r_{c(i)}-S_{i+1} r_{c(i+1)}\right)$

onde, $r_{c}=r_{h} /(a \Delta z)$. 
$\mathrm{Na}$ forma matricial equivalente para a equação 7, desenvolvida abaixo para o conjunto de todas as camadas, as seguintes relações são usadas:

$$
\begin{gathered}
r_{z 1, z 2}=\int_{z_{1}}^{z_{z}} \frac{d z}{K_{z}}=\frac{z_{2}-z_{1}}{K_{e q}} \\
R_{i}=\int_{z_{i}}^{z_{z}} \frac{d z}{K_{z}}=\sum_{j=i}^{n-1} \frac{\Delta z}{K_{j}}+\int_{z_{i}}^{z_{x}} \frac{d z}{K_{z}} \\
r_{i}=R_{i}-R_{i+1}
\end{gathered}
$$



\section{Forma matricial da equação 7}

Essa relação matricial já é suficiente para tratar as fontes e os fluxos de calor sensível e buscar as soluções por métodos iterativos. Porém, quando se trata de desenvolver soluções explícitas; é mais conveniente multiplicar todos os termos dessa equação matricial pela matriz auxiliar dada abaixo (transformação linear) e eliminar o vetor $[\Theta]$ (usando a equação 4) para alcançar finalmente a relação $S=S\left(\Theta_{s}\right)$. A matriz auxiliar e o sistema transformado são dados por,

Matriz Auxiliar $\quad\left[\begin{array}{ccccc}1 & 1 & 1 & 1 & 1 \\ -1 & 1 & 1 & 1 & 1 \\ -1 & -1 & 1 & 1 & 1 \\ -1 & -1 & -1 & 1 & 1 \\ -1 & -1 & -1 & -1 & 1\end{array}\right]$

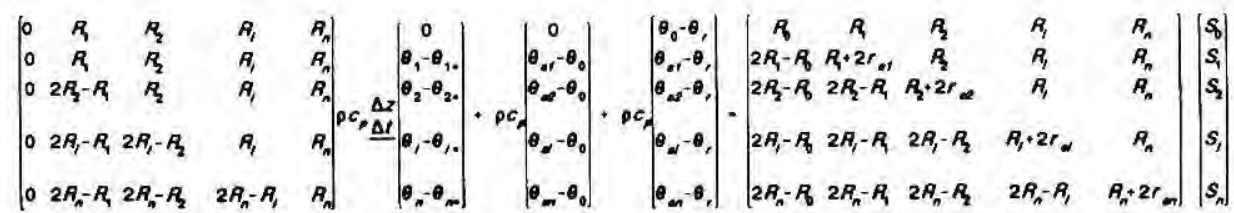

Esta equação pode ser manipulada para evidenciar a relação direta $S=$ $\mathrm{S}\left(\Theta_{\mathrm{s}}\right)$ que sob notação simplificada é dada por, 


$$
[S]=\left[P_{s}\right]\left[\Theta_{s}\right]+\left[Q_{s}\right]
$$

$$
\left[P_{s}\right]=\left[\left[R+r_{c}\right]+[R] \frac{\Delta z}{\Delta t}\left[r_{c}\right]\right]^{-1} p c_{p}\left[[R] \frac{\Delta z}{\Delta t}+2[U]\right]
$$

$$
\left[Q_{s}\right]=\left[\left[R+r_{c}\right]+[R] \frac{\Delta z}{\Delta t}\left[r_{c}\right]\right]^{-1} \rho c_{p}\left[-[R] \frac{\Delta z}{\Delta t}\left[\theta_{t-\Delta t}\right]-\left[\theta_{0}+\theta_{r}\right]\right]
$$

Um procedimento inteiramente similar deve ser adotado no desenvolvimento da equação 2 , associada às concentrações de vapor d'água no ar; em analogia ao fluxo de calor sensível, a seguinte relação pode ser escrita para a concentração de vapor,

$$
\sum_{j=1}^{i} L_{v} \frac{C_{j(t)}-C_{j(t-\Delta t)}}{\Delta t} \Delta z-K_{i} L_{v} \frac{C_{s(i+1)}-C_{s(i)}}{\Delta z}=\left|\sum_{j=0}^{i} V_{j}\right|+\frac{K_{i}}{\Delta z}\left[V_{i} r_{d(i)}-V_{i+1} r_{d(i+1)}\right.
$$

onde, $r_{d}=\left(r_{h}+r_{s}\right) /(a \cdot \Delta z)$.

Neste caso, o desenvolvimento de uma relação direta entre as fontes de vapor d'água e as temperaturas dos elementos vegetais $\left(\mathrm{V}=\mathrm{V}\left(\Theta_{\mathrm{s}}\right)\right)$ passa por uma linearização prévia das concentrações de vapor d'água na superfície dos elementos vegetais (concentração de saturação) em função das temperaturas das superfícies vegetais. Como resultado dessa operação, os termos que são dependentes das concentrações de vapor d'água no ar são também eliminados da equação 9. As duas equações que se seguem mostram esse tipo de linearização.

$$
\begin{aligned}
& L_{v}\left(C_{i+1}-C_{i}\right)=\frac{L_{v} M_{v}}{R^{*} T} \frac{\partial e_{s}}{\partial T}\left(\Theta_{s(i+1)}-\Theta_{s(i)}\right)-V_{i+1} r_{d(i+1)}+V_{i} I_{d(i)} \\
& L_{v} C_{i}=L_{2}\left(\Theta_{s(i)}-\Theta_{1}\right), \frac{L_{v} M_{v}}{R^{\top} T} e_{s}\left(T_{1}\right)-V_{i} r_{d(i)}
\end{aligned}
$$

$T_{1}$ sendo uma temperatura média observada $(\mathrm{To}+\mathrm{Tr}) / 2$.

As correções marginais ligadas ao uso de temperaturas potenciais foram omitidas nessas equações para tornar mais clara a leitura, mas em realidade elas são consideradas na aplicação do modelo. 
A equação matricial para o caso da concentração de vapor d'água apresenta-se da seguinte forma.

$$
\begin{gathered}
{[V]=\left[P_{v}\right]\left[\Theta_{s}\right]+\left[Q_{v}\right]} \\
{\left[P_{v}\right]=\left[\left[R+r_{d}\right]+[R] \frac{\Delta z}{\Delta t}\left[r_{d}\right]\right]^{-1} L_{2}\left[-[R] \frac{\Delta z}{\Delta t}+2[U]\right]} \\
{\left[Q_{v}\right]=\left[\left[R+r_{d}\right]+[R] \frac{\Delta z}{\Delta t}\left[r_{d}\right]\right]^{-1}\left\{[R] \frac{\Delta z}{\Delta t}\left[L_{v}\left[C_{d}\left(T_{1}\right)-C_{t-\Delta t}\right]+L_{2}\left[\theta_{1}\right]\right]-L_{2}\left[\theta_{0}+\theta_{r}\right]+L_{v}[\delta C]\right\}} \\
\text { onde, } L_{2}=\left(\mathrm{L}_{v} M_{v} / R^{*} T\right)(\partial \mathrm{e} / \partial T) .
\end{gathered}
$$

As composições precisas das matrizes individuais que aparecem nas equações 8 e 11 são apresentadas em anexo.

É assumido aqui (detalhes na seção sobre radiação de onda longa) que uma relação similar pode ser obtida para expressar o balanço de energia das superfícies vegetais como uma função das temperaturas dos elementos vegetais, ou formalmente,

$$
\left[\Delta F_{1}\right]=\left[P_{1}\right]\left[\Theta_{s}\right]+\left[Q_{1}\right]
$$

onde, o vetor $\left[F_{1}\right]$ representa para cada camada o balanço de energia em termos da radiação originada nas superfícies dos elementos vegetais e do solo; esse vetor será uma função da distribuição no espaço, das temperaturas médias e do coeficiente de emissão (radiação de onda longa) dos elementos vegetais e do solo entre outros fatores representativos da vegetação.

Desta forma, as equações 8,11 e 12 definem as fontes e a radiação de superfície como elementos internos no sistema solo-planta-atmosfera. Se a variação de energia estocada nos elementos vegetais é considerada pequena numa primeira aproximação então a equação 3 pode ser resolvida, originando uma expressão que determina o vetor das temperaturas dos elementos vegetais (temperatura média das superfícies vegetais existentes em cada camada horizontal),

$$
\left[\Theta_{s}\right]=\left[\left[P_{s}\right]+\left[P_{v}\right]-\left[P_{1}\right]\right]^{-1}\left[\left[\Delta\left(F_{n}-G\right)\right]+\left[Q_{1}\right]-\left[Q_{s}\right]-\left[Q_{v}\right]\right]
$$

\section{Cálculo dos termos de radiação e difusividades turbulentas}

\section{A) Radiação Solar e Atmosférica}

A interação da radiação solar (radiação atmosférica, radiação de elementos vegetais, etc.) com vegetação natural ou modificada é tratada aqui em conformidade com uma teoria geral de transferências radiativas em meio vegetal, 
desenvolvida inicialmente pelos pesquisadores soviéticos nas décadas de $60 \mathrm{e}$ 70. A origem desta teoria reside na adaptação das equações diferenciais expressando essas transferências em meios túrbidos. A turbidez no meio vegetal seria representada pelos elementos vegetais individuais - assimilados à pequenas superfícies planas que refletem, absorvem e transmitem a radiação incidente. Uma síntese desses estudos foi apresentada por ROSS (1981). No contexto das soluçōes analíticas MARQUES FILHO (1992a) propos uma expressão generalizada para descrever o regime de radiação solar no interior da vegetação, separando-o em quatro termos distintos: radiação primária e complementar, cada uma delas tendo componentes direta e difusa. No presente trabalho, adota-se um caso particular dessas soluções, no qual a função que representa a distribuição de inclinações das superfícies vegetais é considerada aleatória, para desenvolver as diferentes componentes de radiação no interior de floresta. Nessa situação, os perfis de radiação solar são descritos pelas seguintes equações:

\section{Radiação Solar Direta}

$$
F_{s}(z)=F_{s}(h) e^{-\frac{A(z)}{2 \cos \theta}}
$$

onde,

$$
\begin{array}{r}
F_{s}(h)-I_{s}(h) \cdot \cos \theta_{s} \\
A(z)-\int_{z}^{h} a(z) d z
\end{array}
$$

\section{Radiação Solar Difusa}

$$
F_{d}(z)=F_{d}(h) .2 E_{3}\left(\frac{A(z)}{2}\right)
$$

onde,

$$
E_{n}(x)=\int_{1}^{\infty} \frac{e^{-x u}}{u^{n}} d u
$$




\section{Radiação Solar Direta Complementar (descendente e ascendente)}

$$
\begin{gathered}
F_{s 1}(A)=\mathrm{K}_{1} F_{s}(A)-\mathrm{K}_{1} \frac{\Phi\left[\mu, r\left(A_{0}-A\right)\right]}{\Phi\left[\mu, r\left(A_{0}\right)\right]} F_{s}(0)-\mathrm{K}_{2} \frac{\Phi[1, r A]}{\Phi\left[\mu, r\left(A_{0}\right)\right]} F_{s}\left(A_{0}\right) \\
F_{s 2}(A)=\mathrm{K}_{2} F_{s}(A)-\mathrm{K}_{1} \frac{\Phi\left[1, r\left(A_{0}-A\right)\right]}{\Phi\left[\mu, r\left(A_{0}\right)\right]} F_{s}(0)-\mathrm{K}_{2} \frac{\Phi[\mu, r A]}{\Phi\left[\mu, r\left(A_{0}\right)\right]} F_{s}\left(A_{0}\right) \\
\mathrm{K}_{i}=\frac{\kappa_{i}}{F_{s}(A=0)} \quad \text { e, } \quad \Phi[x, y]=x e^{y}-(1 / x) e^{-y} .
\end{gathered}
$$

\section{Radiação Solar Difusa Complementar (descendente e ascendente)}

$$
F_{d x}(A)=F_{1}(A)-\frac{\Phi\left[\mu, r\left(A_{0}-A\right)\right]}{\Phi\left[\mu, \Gamma\left(A_{0}\right)\right]} F_{1}(0)-\frac{\Phi[1, r A]}{\Phi\left[\mu, r\left(A_{0}\right)\right]} F_{2}\left(A_{0}\right)
$$

$$
F_{\mathrm{d} 2}(A)=F_{2}(A)-\frac{\Phi\left[1, r\left(A_{0}-A\right)\right]}{\Phi\left[\mu, \Gamma\left(A_{0}\right)\right]} F_{1}(0)-\frac{\Phi[\mu, r A]}{\Phi\left[\mu, r\left(A_{0}\right)\right]} F_{2}\left(A_{0}\right)
$$

As funções e os coeficientes que aparecem nas equações anteriores foram detalhadas no estudo de MARQUES FILHO (1992a).

No mesmo contexto de transferência radiativa em meio vegetal podem ser estudadas a radiação atmosférica, outra fonte de energia exterior ao sistema soloplanta-atmosfera, e o săldo de radiação climático, que são definidos pelas seguintes equações:

$$
F_{d}(z)=F_{a}(h) \cdot 2 E_{3}\left(\frac{A(z)}{2}\right)
$$

$F_{s}(z)-G=\downarrow F_{s}(z)+\downarrow F_{d}(z)+\downarrow F_{s 1}(z)-T F_{s 2}(z)+\downarrow F_{d 1}(z)-T F_{d 2}(z)+\downarrow F_{a}(z)-G$

\section{B) Radiação de Superfície}

A radiação de onda longa das superfícies vegetais e do solo é abordada para o caso em que os elementos da folhagem sejam distribuídos densa e aleatoriamente no espaço. 
Tomando-se uma superfície horizontal como referência no nível z no interior da cobertura vegetal, existem duas situações possíveis para a localização dos elementos vegetais: elementos situados entre o topo da vegetação e a superfície de referência no nível z; ou então elementos que estão entre a referência horizontal e a superfície do solo. Os elementos de folhagem da primeira classe contribuem para a superfície de referência com radiação de onda longa descendente, enquanto que os outros elementos estão associados com radiação ascendente. Esses dois tipos de radiação são descritos em termos de fluxos pelas equações (MARQUES FILHO, 1991):

$$
\begin{aligned}
& \downarrow F(z)=\int_{z}^{h} \epsilon \sigma T_{s}^{4}(y) a(y) E_{2}\left(\frac{A(z)-A(y)}{2}\right) d y \\
& \uparrow F(z)=\int_{0}^{z} \epsilon \sigma T_{s}^{4}(y) a(y) E_{2}\left(\frac{A(y)-A(z)}{2}\right) d y
\end{aligned}
$$

As discretizações apropriadas para essas equações são dadas pelas expressões,

$$
\begin{aligned}
& \downarrow F\left(z_{i}-\Delta z / 2\right)=\sum_{j=i}^{N} \epsilon \sigma T_{s}^{4}(j) a(j) E_{2}\left(\frac{A\left(z_{i}-\Delta z / 2\right)-A\left(z_{j}\right)}{2}\right) \Delta z \\
& \uparrow F\left(z_{i}+\Delta z / 2\right)=\sum_{j=1}^{i} \epsilon \sigma T_{s}^{4}(j) a(j) E_{2}\left(\frac{A\left(z_{j}\right)-A\left(z_{i}+\Delta z / 2\right)}{2}\right) \Delta z
\end{aligned}
$$

A figura I ilustra o caráter mutual de interação da radiação das superfícies vegetais; os coeficientes de atenuação A e B estão associados às exponenciais integrais e são dados pelas equações:

$$
\begin{aligned}
& A_{i-1, j}=E_{2}\left(\frac{A\left(z_{i}-\Delta z / 2\right)-A\left(z_{j}\right)}{2}\right) \\
& B_{i, j}=E_{2}\left(\frac{A\left(z_{j}\right)-A\left(z_{i}+\Delta z / 2\right)}{2}\right)
\end{aligned}
$$




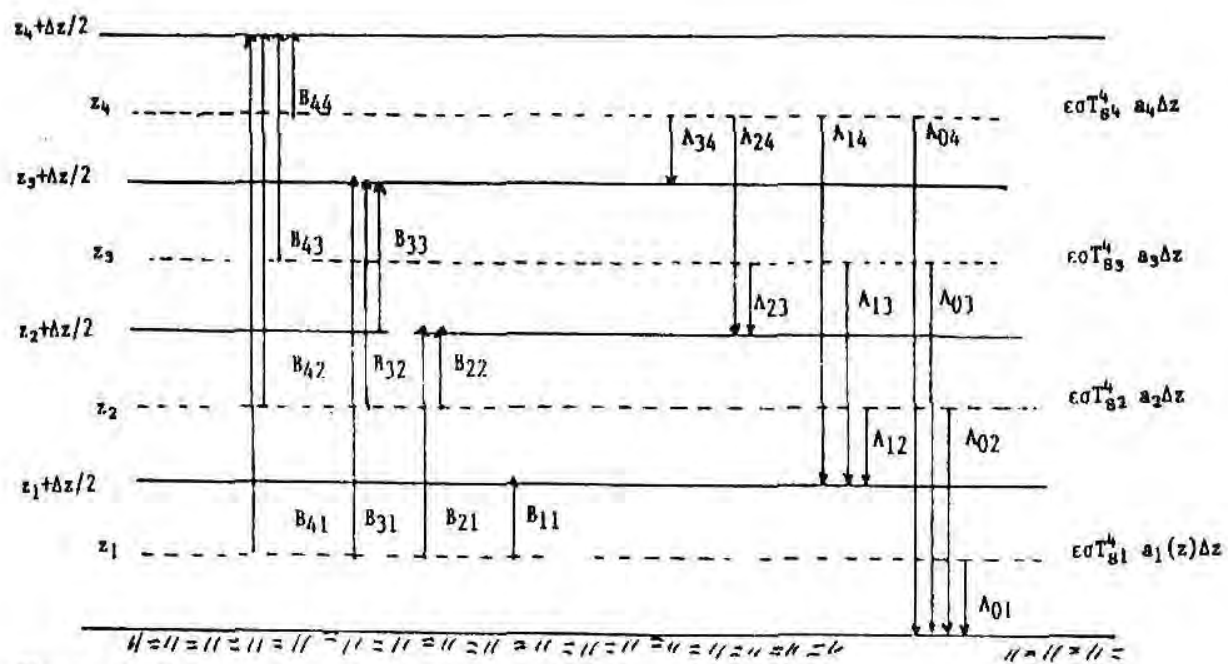

Figura 1. Radiação de onda longa das superfícies vegetais.

A forma matricial equivalente às equações 24 e 25 , incluindo uma formulação para a radiação do solo, é dada pela expressão,

$\left[\begin{array}{l}F_{L O} \\ \Delta F_{L 1} \\ \Delta F_{L 2} \\ \Delta F_{L i} \\ \Delta F_{L n}\end{array}\right]=\left[\begin{array}{ccccc}-1 & A_{0,1} & A_{0,2} & A_{0, j} & A_{0, n} \\ 1-\alpha_{1} & -B_{1,1}-A_{0,1} & A_{1,2}-A_{0,2} & A_{1, j}-A_{0, j} & A_{1, n}-A_{0, n} \\ \alpha_{1}-\alpha_{2} & B_{1,1}-B_{2,1} & -B_{2,2}-A_{1,2} & A_{2, j}-A_{1, j} & A_{2, n}-A_{1, n} \\ \alpha_{i-1}-\alpha_{i} & B_{i-1,1}-B_{i, 1} & B_{i-1,2}-B_{i, 2} & -B_{i, j}-A_{i-1, j} & A_{i, n}-A_{i-1, n} \\ \alpha_{n-1}-\alpha_{n} & B_{n-1,1}-B_{n, 1} & B_{n-1,2}-B_{n, 2} & B_{n-1, j}-B_{n, j} & -B_{n, n}-A_{n-1, n}\end{array}\right]\left[\begin{array}{c}E_{M 0} \\ E_{M 1} \\ E_{M 2} \\ E_{M i} \\ E_{M n}\end{array}\right]$

\section{Radiação de onda longa das superfícies vegetais e do solo em forma matricial}

Os coeficientes que aparecem nesta configuração matricial representam a atenuação da radiação do solo. que pode ser calculada como um caso particular da equação de transferência radiativa dado por:

$$
\begin{aligned}
& \uparrow F_{\text {solo }}(z)=\epsilon \sigma T_{s}^{4}(0) \alpha_{\text {solo }}(z) \\
& \alpha_{\text {solo }}(z)=2 E_{3}\left(\frac{A(0)-A(z)}{2}\right)
\end{aligned}
$$

A radiação emitida pelos elementos vegetais em cada camada horizontal ou pela superfície do solo $\left(\mathrm{E}_{\mathrm{Mi}}=\varepsilon \sigma \mathrm{T}_{\mathrm{si}}{ }^{4} \cdot \mathrm{a}_{\mathrm{i}} \cdot \Delta \mathrm{z}\right)$ são linearizadas em função da 
temperatura $T_{1}$ anteriormente definida sob a seguinte forma:

$$
\epsilon \sigma T_{s i}^{4}=\epsilon \sigma T_{1}^{4}+4 \epsilon \sigma T_{1}^{3}\left(T_{s i}-T_{1}\right)
$$

A adaptação desta equação para a forma da expressão 12 permite desenvolver as seguintes igualdades (as correções marginais ligadas à temperaturas potenciais são consideradas no modelo embora não apareçam no texto):

$$
\begin{gathered}
{\left[\Delta F_{1}\right]=\left[P_{1}\right]\left[\Theta_{s}\right]+\left[Q_{1}\right]} \\
{\left[P_{1}\right]=[A B]\left[a_{1} \cdot \Delta z\right] \cdot 4 \epsilon \sigma T_{1}^{3}} \\
{\left[Q_{1}\right]=[A B]\left[a_{1} \cdot \Delta z\right] \cdot\left(-3 \epsilon \sigma T_{1}^{4}\right)}
\end{gathered}
$$

C) Difusividades Turbulentas, resistências de camada limite e estomática

É assumido que as difusividades turbulentas intervenientes na formação dos fluxos de vapor d'água e de calor sensível possam ser associadas ou determinadas a partir das difusividades relacionadas aos fluxos de quantidade de movimento. Nessas condições, um procedimento baseado sobre a Teoria de Monin-Obukhov pode ser usado para descrever as difusividades sobre a vegetação pelas seguintes equações:

$$
\begin{gathered}
K_{v, s}=\frac{\kappa(z-d) u}{\phi_{v, s}\left(\frac{z-d}{L}\right)} \\
K_{V, s}=\frac{K_{m} \cdot \phi_{m}\left(\frac{z-d}{L}\right)}{\phi_{V, s}\left(\frac{z-d}{L}\right)}
\end{gathered}
$$

No interior da vegetação a absorção de quantidade de movimento pelos elementos vegetais regula e determina a forma dos perfis de velocidade de vento. Tal absorção é controlada pela equação diferencial,

$$
K_{M} \frac{\partial^{2} u}{\partial z^{2}}+\frac{\partial K_{M}}{\partial z} \frac{\partial u}{\partial z}-c_{d}(z) a(z) u^{2}=0
$$


A hipótese de similaridade entre os perfis de velocidade do vento e das difusividades turbulentas (COWAN (1968)) é generalizada aqui, considerandose que tal similaridade é afetada por uma função $\eta(z)$, que pode ser produzida a partir de argumentos teóricos ou experimentais e expressa diferentes aspectos estruturais da vegetação. A equação correspondente é dada por ,

$$
\frac{K_{M}(z)}{K_{M}(h)}=\frac{u(z)}{u(h)} \eta(z)
$$

Combinando essas duas equações, obtem-se uma equação diferencial de segunda ordem homogênea,

$$
u \frac{\partial^{2} u}{\partial z^{2}}+\frac{\eta^{\prime}}{\eta} u \frac{\partial u}{\partial z}+\left[\frac{\partial u}{\partial z}\right]^{2}-\lambda^{2} u^{2}=0
$$

onde,

$$
\lambda^{2}(z)=\frac{c_{d}(z) a(z) u(h)}{\eta(z) K_{\underline{N}}(h)}
$$

A solução desta equação diferencial deve ser obtida inicialmente através de uma redução de ordem pela transformação de variável,

$$
u(z)=e^{\int p_{0}(z) d z}
$$

$\mathrm{p}_{\mathrm{i}}$ (z) sendo uma nova variável descrita por uma equação de primeira ordem. Por esse procedimento e adotando-se as condições limites,

$$
\begin{array}{ll}
\mathrm{z}=0 & \mathrm{u}=0 \\
\mathrm{z}=\mathrm{h} & \mathrm{u}=\mathrm{u}_{\mathrm{h}},
\end{array}
$$

a solução da equação foi desenvolvida sob a forma,

$$
K_{V, s}(z)=K_{V, s}(h) e^{-\int_{:}^{h} p(z) d z} \sqrt{\frac{\int_{0}^{z} e^{-\int 4 p(z) d z} \cdot\left(\frac{1}{\eta(z)}\right) d z}{\int_{0}^{h} e^{-\int 4 p(z) d z} \cdot\left(\frac{1}{\eta(z)}\right) d z}}
$$

onde p(z) é uma solução particular de uma equação não-linear de primeira ordem (MARQUES FILHO,1992b).

As resistências de camada limite são calculadas como funções de parâmetros adimensionais, tais como: Número de Reynolds, $\mathrm{R}_{\mathrm{e}}$, número de Schimdt, $S_{\mathfrak{v}}$, e em conformidade às discussões sobre os modelos envolvidos 
apresentadas por BRUTSAERT (1979):

$$
\begin{gathered}
\frac{1}{C_{t} u}=r_{b(v, s)}=\frac{C t e}{u} R_{e}^{m} S_{c}^{n} \\
r_{b(v)}=0,92 \cdot r_{b(s)}=\frac{0,91}{u} S_{c}^{2 / 3} R_{e}^{1 / 2}
\end{gathered}
$$

No domínio relativo ao controle dos elementos vegetais sobre os fluxos, as resistências estomáticas em cada camada no interior da vegetação são calculadas como funções da radiação PAR, temperatura, e déficit de saturação do ar, organizadas na seguinte equação:

$$
r_{s}=r_{s 1}\left(F_{P A R}\right) r_{s 2}(T) r_{s 3}(\delta q)
$$

As funções particulares que aparecem na expressão apresentada acima são definidas de forma similar aos trabalhos de SHUTTLEWORTH (1989) e SELLERS et al. (1989). Por exemplo, a função $r_{\mathrm{s} 1}$ é calculada em relação à radiação PAR pela equação,

$$
r_{s 1}\left(F_{P A R}\right)=\left(\frac{a}{F_{P A R}}+b\right) \cdot c
$$

onde, os coeficientes assumem os seguintes valores: $a=2336 ; b=154 ; c=1,1$. Uma estimativa para o fluxo $\mathrm{F}_{\text {par }}$ pode ser feita com base nos perfis de radiação solar modelizados no presente trabalho, resultando aproximadamente a relação $\mathrm{F}_{\text {par }}=\mathrm{F}_{\mathrm{g}} / 4, \mathrm{~F}_{\mathrm{g}}$ sendo a radiação solar global em cada camada da vegetação (MARQLUES ${ }^{\prime \prime}$ FILHO, 1991).

\section{APLICAÇÃO DO MODELO}

Medidas micrometeorológicas realizadas acima e no interior de floresta na Amazonia (Reserva Florestal Ducke, Manaus, AM) (SHUTTLEWORTH et al., 1984a, b, 1985) são usadas para testar o conjunto de modelos aqui desenvolvidos. O sistema de aquisição de dados é composto por instrumentos para medir e registrar os fluxos de vapor d'água, de calor sensível e de quantidade de movimento acima da vegetação; perfis de temperatura e umidade do ar, velocidade do vento acima e no interior da cobertura vegetal; medidas no domínio da fisiologia vegetal complementam o conjunto de informações.

A distribuição vertical da folhagem para a floresta da Reserva Dcuke é mostrada na figura 2 (Marques Filho, 1991). O LAI para esta vegetação densa é assumido como sendo aproximadamente 8 . As simulações que se seguem são pouco sensíveis ao valor exato do LAI, notadamente na faixa de valores entre 6 e 10 . 


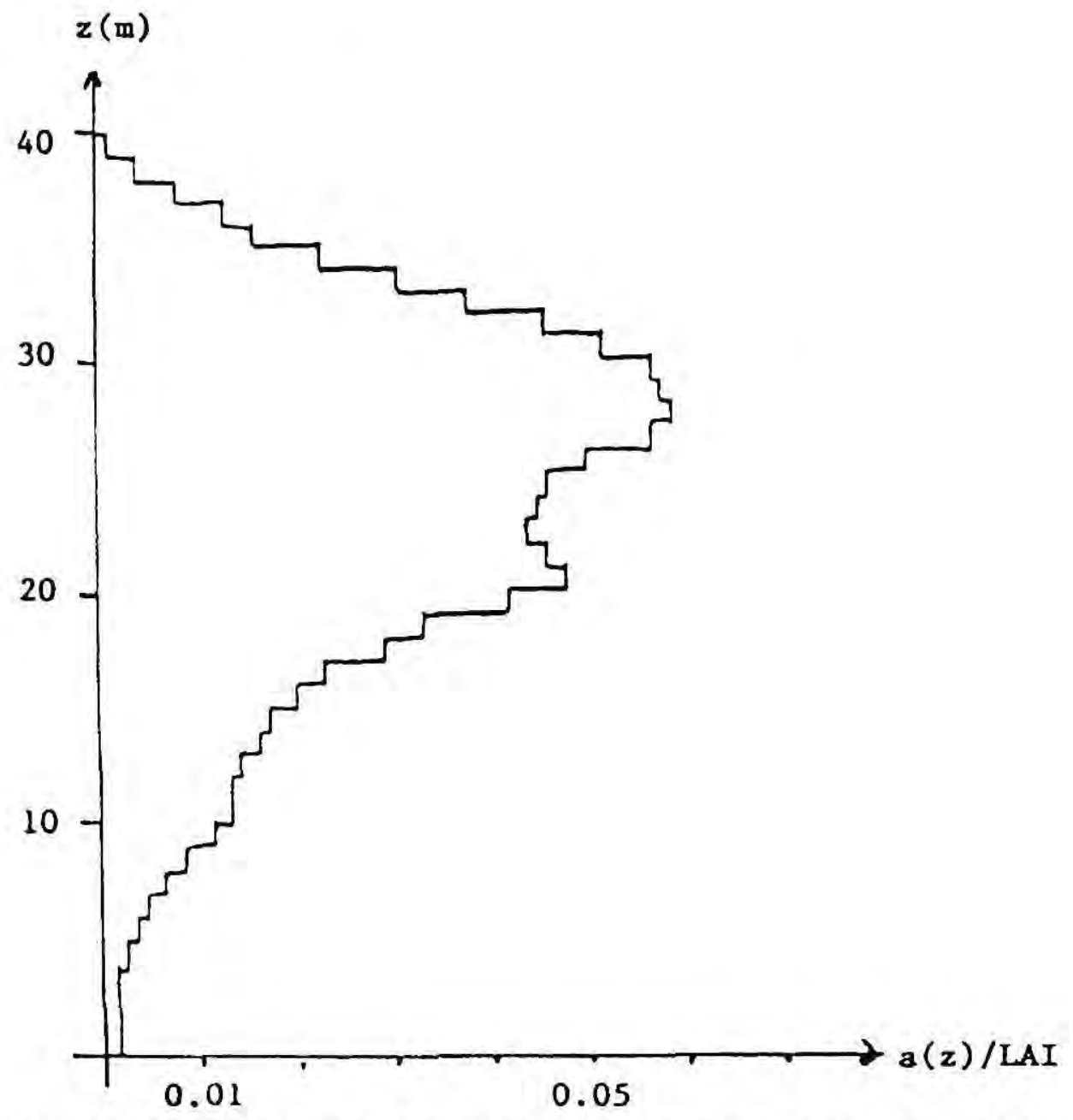

Figura 2. Distribuição de área foliar na vertical para a floresta da Reserva Ducke (Marques Filho, 1991).

As figuras 3 e 4 correspondentes aos dias 21 e 23 de agosto de 1984 proporcionam uma primeira comparação entre os fluxos calculados pelo modelo e os fluxos medidos no topo da vegetação (vapor d'água, calor sensível) pelo HYDRA (descrição do sistema, SHUTTLEWORTH et al. 1982). Ocorre uma concordância aceitável em termos gerais entre valores calculados (curvas pontilhadas) e medidos (curvas contínuas), as diferenças sendo mais importantes nas horas que apresentam uma forte variação da radiação global. Nessas ocasiões, os resultados produzidos pelo modelo são bastante concordantes e seguem de perto as flutuações da radiação; este não é o caso das medidas produzidas pelo sistema Hydra, um evento factual que está provavelmente associado aos procedimentos internos do sistema para o cálculo dos fluxos. 


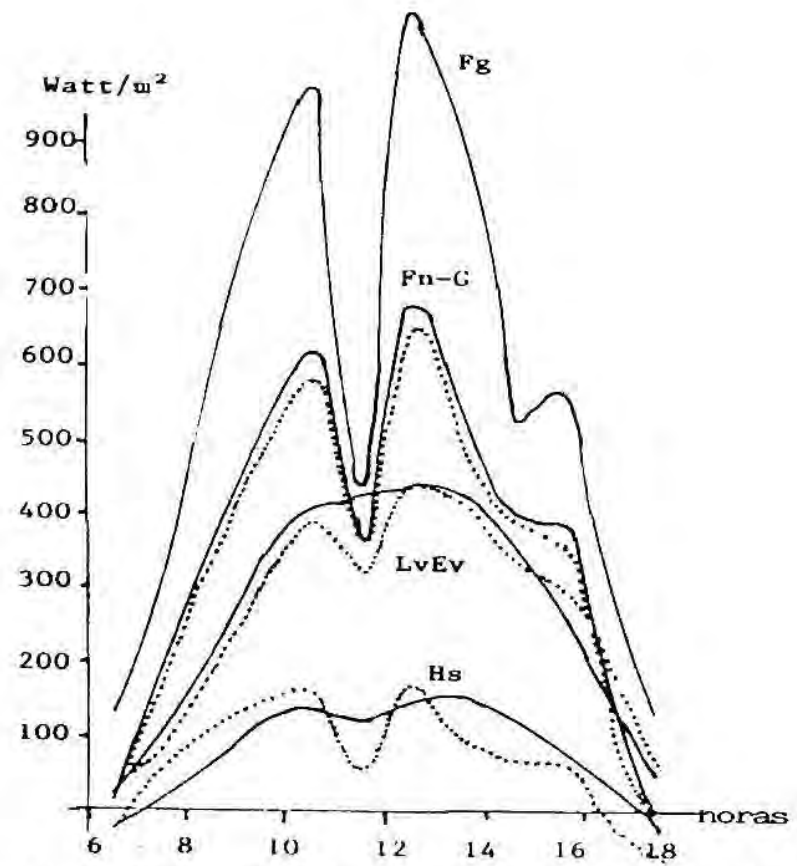

Figura 3. Comparação dos fluxos calculados e observados no topo da vegetação (Floresta Amazônica - estação seca - 21/08/84)

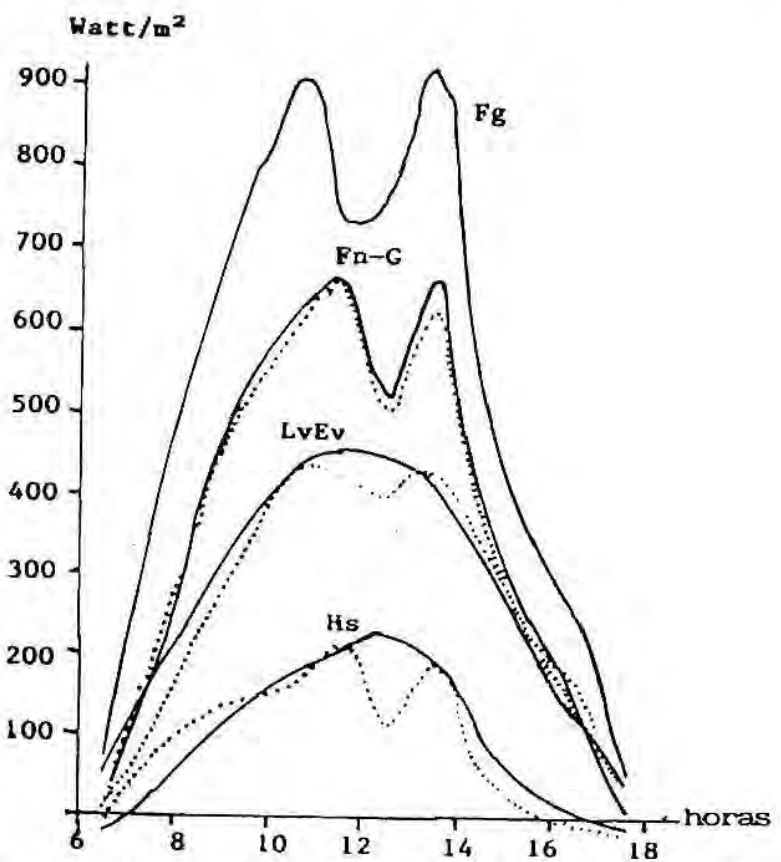

Figura 4. Comparação dos fluxos calculados e obscrvados no topo da vegetação (Floresta Amazônica - estação seca - 23/08/84) 
Na figura 5 é mostrada a modelização obtida para um dia completo (0$24 \mathrm{~h} ; 14 / 4 / 1985$ ). As curvas modelizadas e observadas são muito próximas entre si, excetuando-se o caso do fluxo de calor sensível que apresenta uma discrepância maior no início da manhã.

De um modo geral nessas três figuras pode-se verificar que a modelização dos fluxos de vapor d'água é superior àquela obtida para o fluxo de calor

Watts $/ \mathrm{m}^{2}$

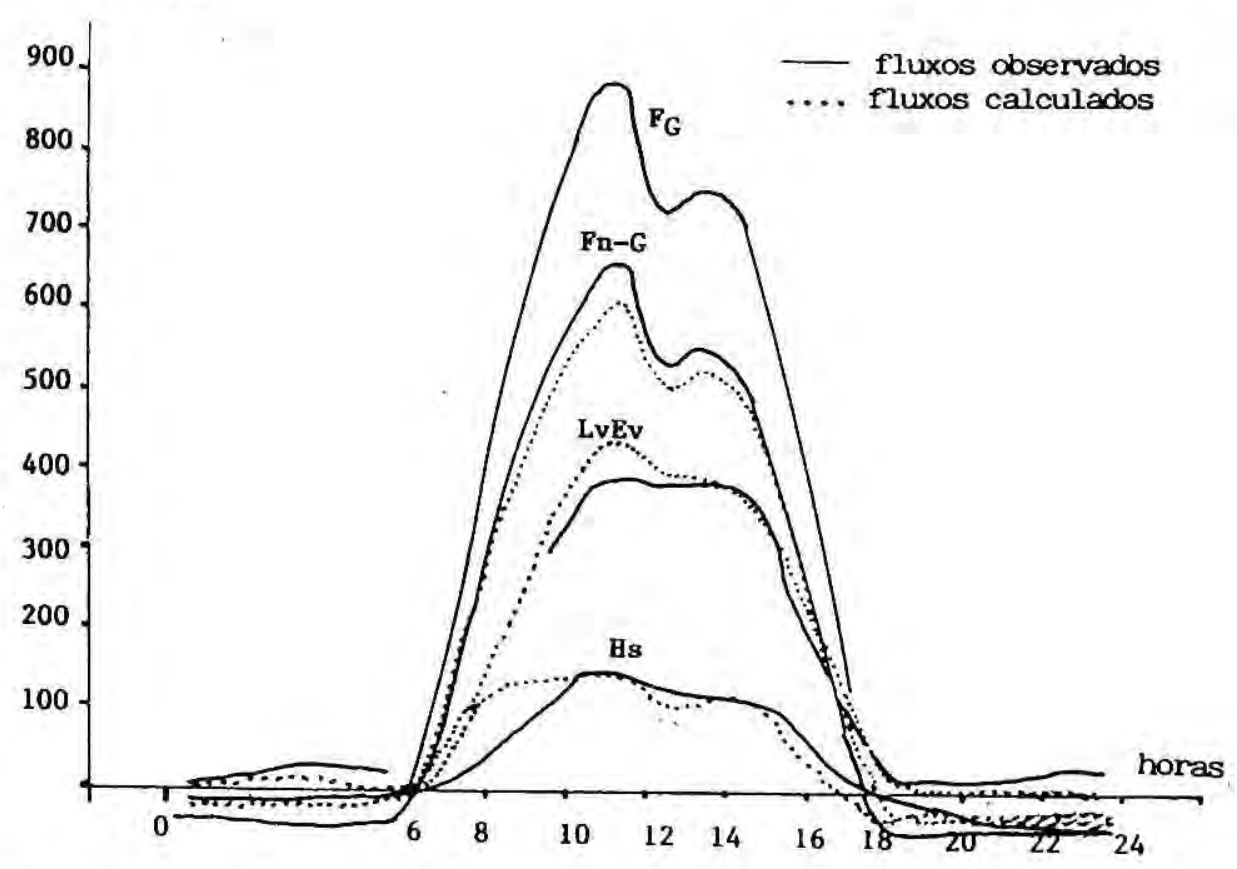

Figura 5. Fluxos Calculados e Observados sobre a Floresta Amazônica (14/04/85)

sensível; em relação às curvas obtidas para o saldo de radiação, a concordância global dá uma indicação sobre a qualidade da modelização da radiação de onda longa, notadamente a radiação dos elementos vegetais.

A distribuição das fontes de energia (vapor d'água e calor sensível) no interior da vegetação são ilustradas pelas figuras 6 e 7, que em realidade mostram a evolução temporal no período diurno do dia 14/04/1985. Existe uma certa relação ao menos em termos qualitativos entre a distribuição das fontes de vapor d'água e a distribuição de folhagem na vertical (figura 2), fato que revela a importância desta característica da vegetação no processo de evaporação. As fontes de calor sensível não apresentam a mesma dependência. Em contrapartida, os efeitos da variação temporal da radiação global no topo da vegetação são claramente propagados e influenciam as formas das curvas associadas a essas fontes no interior da vegetação. 

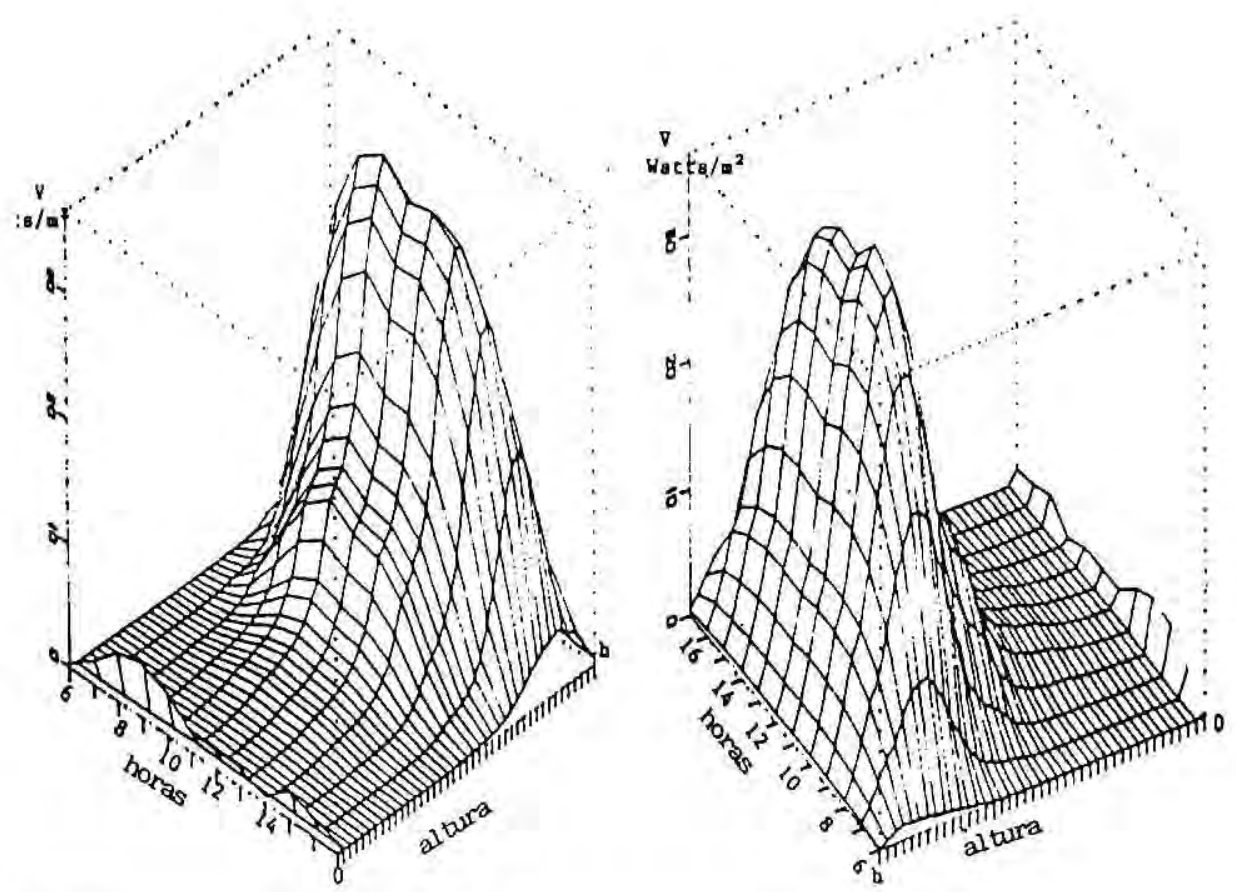

Figuras 6a et 6b. Fontes de vapor d'água no interior da vegetação,
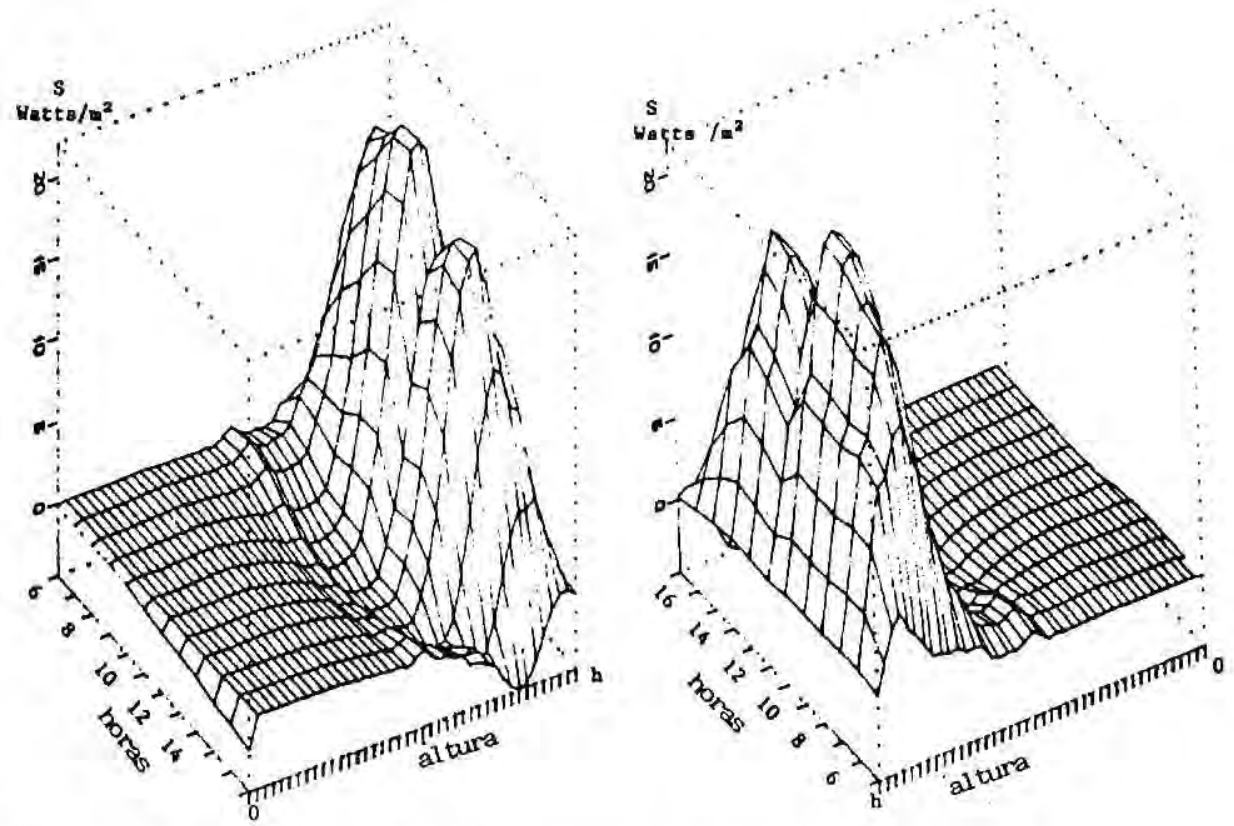

Figuras 7a et $7 \mathbf{b}$. Fontes de calor sensível no interior da vegetação. 
A evolução temporal dos perfis de temperatura e umidade do ar é representada nas figuras 8 e 9 , respectivamente, em confrontação com os perfis medidos acima e entre os elementos vegetais da floresta.

As funções adimensionais de estabilidade que aparecem nas equações relativas às difusividades turbulentas (equações 26 e 27 foram desenvolvidas a partir de medidas simultâneas de fluxos e gradientes sobre a floresta (MARQUES FILHO \& RIBEIRO, 1987). Elas diferem das funções existentes na literatura e são calculadas pelas seguintes equaçôes (atmosfera instável):

$$
\begin{aligned}
& \phi_{m}=\left(1-36 \frac{z-d}{L}\right)^{-2 / 9} \\
& \phi_{S, v}=\left(1-48 \frac{z-d}{L}\right)^{-3 / 5}
\end{aligned}
$$

A concordância entre os perfis calculados e observados de temperatura do ar é aceitável, com distorções mais importantes ao nível da superfície do solo. Nas figuras 8 e 9 estão representadas curvas suplementares que estão associadas a uma versão simplificada do modelo onde as correções de estabilidade não são consideradas.

No caso dos perfis de vapor d'água (umidade específica) a aproximação entre as curvas é menos perfeita, e as discrepâncias crescem bastante quando a versão simplificada do modelo é utilizada, especialmente nos períodos em que os fluxos de calor sensível são elevados (12-14h) e conseqüentemente as correções de estabilidade são significativas. Isto é bem confirmado nos perfis de umidade específica do ar, mas menos perceptível para o caso dos perfis de temperatura do ar. Ainda no caso da aplicação do modelo em sua versão mais desenvolvida restam algumas diferençats entre os perfis. Essas diferenças devem estar associadas alos diferentes-fatores que não foram considerados na versão atual ou que são considerados constantes, como as características do ar, por exemplo (densidade do ar e sua dependência em relação a temperatura e umidade).

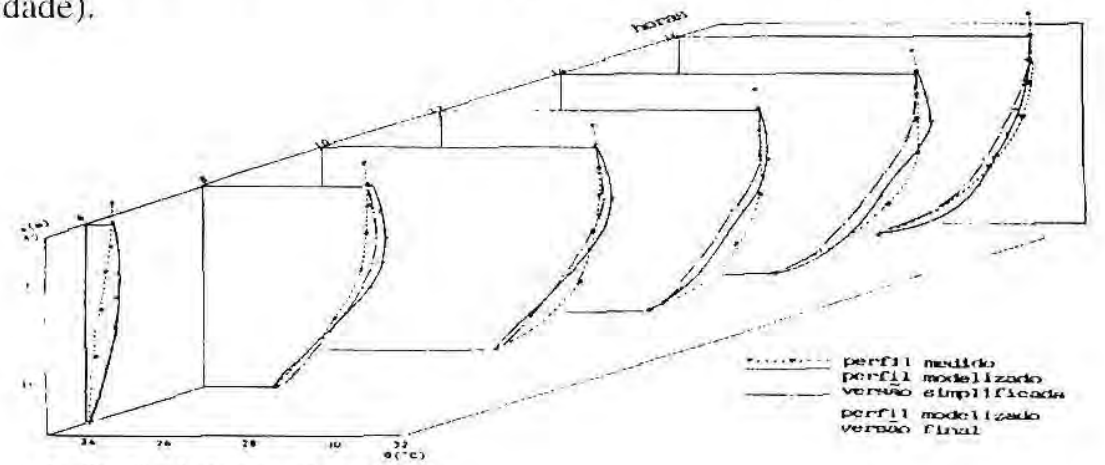

Figura 8. Perfis de temperatura do ar: 


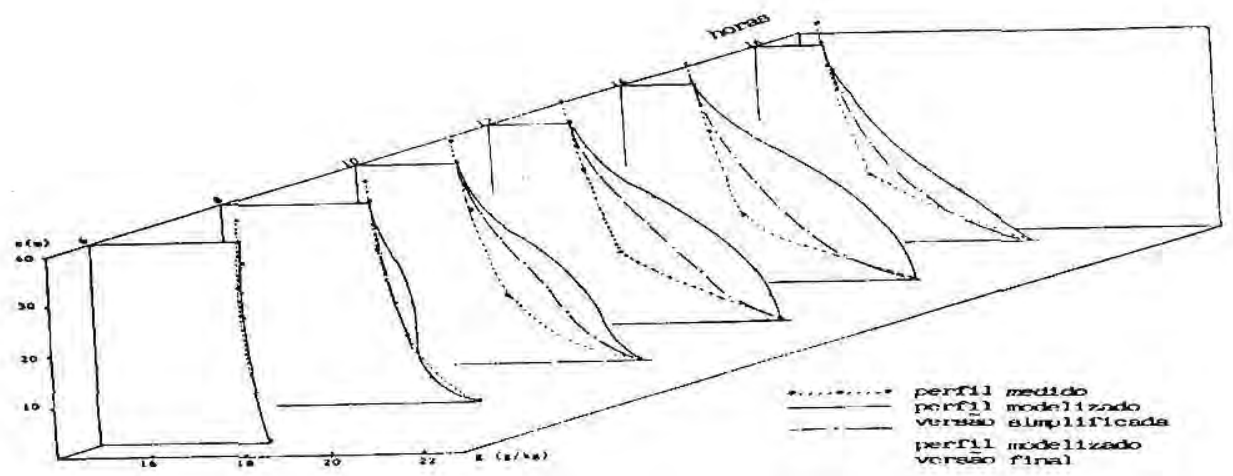

Figura 9. Pertis de umidade do ar.

\section{CONCLUSÃO}

Um modelo geral para descrever as transferências de massa e de energia resultantes da interação entre coberturas vegetais e a atmosfera foi apresentado. Simulações bem aceitáveis dos fluxos de vapor d'água e de calor sensível e dos perfis de temperatura e de umidade específica do ar podem ser obtidas. No interior da vegetação, todos os termos de radiação podem ser determinados, e particularmente um modelo para representar a radiação de onda longa das superfícies vegetais e do solo é apresentado.

A confrontação entre os resultados que são produzidos pela aplicação do modelo para uma floresta da Amazonia e as observações de fluxos e perfis é suficientemente positiva, revelando o realismo da formulação adotada aqui para estudar as trocas entre uma floresta densa e a atmosfera.

As perspectivas para aperfeiçoar tal modelo residem principalmente num tratamento separado para o sistema solo, que pode ser posteriormente conectado ao sistema principal, via entre outras variáveis, a temperatura e umidade da superfície do solo.

\section{AGRADECIMENTOS}

O autor expressa seu reconhecimento ao Professor Alain PERRIER e a sua Assistente Andrée TUZET, pesquisadores do INAPG (Paris - França), pelas valiosas discussões, contribuições e criticismo sobre diferentes aspectos desta análise e pelo suporte recebido ho seio da "Chaire de Bioclimatologie - INSTITUT NATIONAL AGRONOMIQUE PARIS - GRIGNON" no período de setembro de 1988 a agosto de 1991.

\section{Literatura citada}

ABRAMOWITZ, M., STEGUN, 1., 1970 - "A Handbook of Mathematical Functions" Dover Publications Washington D.C. 1046 pgs.

BACHE, D. H., 1986a - "Momentum Transfer to Plant Canopies. Inlluence of Structure and Variable Drag" - Ammospheric Enviromment - vol. 20,n. 7:1369-378.

BACHE, D. H., 1986b - "On the Theory of Gascous Transport to Plant Canopies Atmospheric Enviromment - vol 20, n.7: 1379-388. 
BRUTSAERT, W., 1979 - "Heat and Mass Transfer to and from Surlaces with Dense Vegetation or Similar Permeable Roughness" - Boundary Layer Meteorology 16: 365-388.

CHEN, J., 1984 - "Uncoupled Multi-Layer Model for the Transler of Sensible and Latent Heat Flux Densities from Vegetation" - Bondary Laver Meteorology - 28 : 213-225

COWAN, I.R., 1968a - "Mass, Heat and Momentum Exchange between Stands of Plants and Their Atmospheric Environment" Quart. I. Rosal Me'teroulogy Sox: 94: 523-544.

FURNIVAL, G.M., WAGGONER. P.E., REIFSNYDER. W.E., 1975 - "Computing the Energy Budget of aLeal Canopy with Matrix Algehra and Numerical Integration" - Agricultural Meterorology - 14: $405-416$.

HALLDIN, S.. LINDROTH. A.. 1986 - "Pine Forest Microclimale Simulation Using Different Diffusivities" - Boundary Layer Metesrology 35: 103-123.

MARQUES FILHO, A.O. 1991 - "Bilan inergétique d'unc foret cquatoriale. Modetisation mathamatiglue " thèse de Doctorat (INAPG), Paris, 183 pages.

MARQUES FILHO, A.O. 1992a "Modéles des transferts radiatils à 1 intérieur des couverts végétaux - les solutions analytiques." Acta Amasontica, 22 (4) : $5+1-565$.

MARQUES FILHO, A.O. 1992h "Translents de quantité de mouvement alt-dessus et à intéricur de la végétaltion" Acta Amasonica, $22(4): 567-585$.

MARQUES FILHO, A.O., RIBEIRO, M.N.G., 1987 - "Relações entre lluxos e gradientes sobre llorestas" - Anciis do VII Simposio Brasilerio de Hidrotogia or Rectarsos Hidricos - Salvador.

MASSMAN, W.1. 1987:1 - "A comparative Study of Some Simple Matrematical Models of Mean Wind Structure and Acrodynamic Drag of Plant Canopies" Bosundary Layer Meteonology 40: 179. 197.
MASSMAN, W.J., 1987b - "Heat Transfert to and From Vegetated Surfaces: an Analytical Method for the Bulk Exchange Coefficients" Bentandary Layer Meteorology 40: 1269-281.

MASSMAN, W., VAN DIJKEN, A., 1989 -

"Water Vapor Transter from a Vegetated Surliace: Numerical Study of Bulk Transfer Coclficients and Canopy Resistances" - Bonendary Layer Meteorology 49; 295 307.

MCNEIL, D.D., SHUTTLEWORTH, W.J.. 1975 - "Comparative Measurements of the Energy huxes over a Pine Forest" Bonnckary Laver Meteorology 9: 297-313.

MYNENI, R.R., ROSS, J., ASRAR, G., 1989 "A Review on the Theory of Photon Transpont in Plant Canopies" - Agricultural and Forest Meteorolog! 45: 1-153.

PERRIER, A., 1967 - "Approche Théorique de la Microturbulence et des Transferts dans les Courves Vegetaux en Vue de l'Analyse de la Production Végétale" - La Mútemologice, 5(4):527-550).

PERRIER, A., 1976 - "Etude et Essai de Modatisation des Eidunges de Masse at dinergie au Nivean des Couverts Vegritute" - These de Doctorat d'Etat Université Pierre et Marie Curie. Paris Vl.

PHILIP. J.R.. 1964t - "Sources and Transfer Processes in the Air Layers Occupied by Vegetation" - Tentrat of Applied Meteonology 3: $390-395$.

ROSS, I. 1981 - "The Radiation Regime and the drehitecture of Plant Stands" - Dr. W. Junk Publ,. The Nellerlands.

SELLERS. P. J.. SHUTTLEWORTH, W.J. DORMAN, I.L.. DALCHER, A., ROB. ERTS. J. M. 1989 - "Calibrating the Simple Biosphere Model (SiB) for Amaan clatta. Part I: Average Calibration with field Datil" - . Iournal of Applied Meternmolegy 28: 727-759.

SHUTTLEWORTH, W.J. MCNEIL, D.D. MOORE. C.J., 1982 - "A Switched Continuous-wave Sonic Anemometer lor Measuring Surtace Heat Fluxes Boundary Layer Meteorology 23; 425-448. 
SHUTTLEWORTH, W.J., GASH, J.H.C., LLOYD, C.R., MOORE, C.J., ROBERTS, J.M., MARQUES FILHO, A.O,, FISCH, G.F., SILVA FILHO, V.P., RIBEIRO, M.N.G., MOLVION, L.C.B., ABREU SA, L.D., NOBRE, J.C.A.. CABRAL, O.M.R., PATEL, S.R.. MORAES, J.C., 1984A - "Eddy Corrclation Measurements ol Energy Partition for Amazonian Forest" - Quarl, J.R. Meteorol. Soc: 110: 1143-162.

SHUTTLEWORTH, W.J., GASH, J.H.C., LLOYD, C.R., MOORE. C.J., ROBERTS, J.M., MARQUES FILHO, A.O., FISH, G.F., SILVA FILHO, V.P. RIBEIRO, M.N.G., MOLION, L.C.B.. ABREU SA, L.D.. NOBRE, J.C.A., CABRAL, O.M.R., PATEL,S.R., MORAES, J.C., 1984b - "Ohservations of Radiation Exchange above and below Amazonian Forest" - Quat, I.R. Meteorol. Soc: 110: 1163-1169.

SHUTTLEWORTH, W.J., GASH, J.H.C., LLOYD, C.R., MOORE, C.J., ROBERTS, J.M,, MARQUES FILHO, A.O., FISCH, G.F., SILVA FILHO, V.P., RIBEIRO, M.N.G., MOLION, L.C.B., ABREU SA, L.D., NOBRE, J.C.A., CABRAL, O.M.R., PATEL, S.R., MORAES. J.C., 1985 - "Daily Variations of Temperature and Humidity within and above Amazonian Forest" - Weather 40: 102-108.

TUZET, A., 1989 - Simulation of the Ficld Drying of Hay" - f" International Green Crop? Congres - April 1989 - Cambridge. UK.

VAN DE GRIEND, A.A., VAN BOXEL, J.H., 1989 - "Water and Energy Balance with a Multilayer Canopy Representation for Remote Sensing Purposes" - Water Resources Resecuch - Vol 25 (5): 949-971.

WAGGONER, P.E., REIFSNYDER. W.E., 1968 - "Simulation of Temperature, Humidity and Evaporation Profiles in a Leall Canopy" - Ioumal of Applied Metersology - 7: 400-409.

IVAGGONER. P.E., FURNIVAL. G.M.. REIFSNYDER, W.E., 1969 - "Simulation of the Microclimate in a Forest" - Forest Science. 15(1):37-45

\section{LISTA DOS SÍMBOLOS PRINCIPAIS}

$\mathrm{A}(\mathrm{z}) \rightarrow$ distribuição vertical de área foliar acumulada.

Aij - coeficiente de atenuação de radiação de onda longa descendente (superfícies vegetais).

$a(z)$ - distribuição vertical de área foliar $\left(\mathrm{m}^{2} / \mathrm{m}^{3}\right)$.

Bij - coeficiente de atenuação de radiação de onda longa ascendente (superfícies vegetais).

$\mathrm{C} \rightarrow$ concentração de vapor d'água do ar $\left(\mathrm{kg} / \mathrm{m}^{3}\right)$.

$\mathrm{C}_{\mathrm{s}} \rightarrow$ concentração de saturação em vapor d'água na temperatura da superfície $\left(\mathrm{kg} / \mathrm{m}^{3}\right)$.

$c_{\mathrm{p}} \rightarrow$ calor específico do ar a pressão constante (Joule/( $\left.\mathrm{kg}^{\prime \prime} \mathrm{K}\right)$ ).

$\mathrm{c}_{\mathrm{vg}} \rightarrow$ calor específico médio dos elementos vegetais (Joule/( $\left.\mathrm{kg}{ }^{\prime \prime} \mathrm{K}\right)$ ).

$\mathrm{d} \rightarrow$ altura de deslocamento do plano zero $(\mathrm{m})$.

$\mathrm{E}_{\mathrm{v}}$ - evaporação $\left(\mathrm{kg} /\left(\mathrm{m}^{2} \mathrm{~s}\right)\right)$.

$\mathrm{E}_{n}(\mathrm{x}) \rightarrow$ integral exponencial.

e - pressão de saturação de vapor d'água (Newton $/ \mathrm{m}^{2}$ ).

$\mathrm{F} \rightarrow$ fluxos de radiação $\left(\mathrm{Watt} / \mathrm{m}^{2}\right)$.

$\mathrm{F}_{\mathrm{a}}$ - radiação atmosférica (Watt/m²). $\mathrm{F}_{u} \rightarrow$ radiação solar difusa (Watt $/ \mathrm{m}^{2}$ ).

$\mathrm{F}_{\mathrm{t} 1}{ }^{-}$radiação solar difusa complementar descendente (Watt/ $\mathrm{m}^{2}$ ).

$\mathrm{F}_{\mathrm{d} 12} \rightarrow$ radiação solar difusa complementar ascendente (Wat $\left./ \mathrm{m}^{2}\right)$.

$\mathrm{F}_{y^{-}}$- radiação solar global (Watt $\left./ \mathrm{m}^{2}\right)$.

$\mathrm{F}_{1}$ - radiação de onda longa das superfícies vegetais (Watt/m²). 
$\mathrm{F}_{11} \rightarrow$ radiação de onda longa descendente das superfícies vegetais (Watt $/ \mathrm{m}^{2}$ ).

$\mathrm{F}_{12} \rightarrow$ radiação de onda longa ascendente das superfícies vegetais $\left(\mathrm{Watt} / \mathrm{m}^{2}\right)$.

$\mathrm{F}_{\mathrm{nc}}-$ saldo de radiaçĩo climático (Watt $/ \mathrm{m}^{2}$ ).

$\mathrm{F}_{n} \rightarrow$ saldo de radiação $\left(\mathrm{Watt} / \mathrm{m}^{2}\right)$.

$\mathrm{F}_{*}$ - radiação solar direta (Watt/m²).

$\mathrm{F}_{\mathrm{s} 1}$ - radiação solar direta complementar descendente (Watt $\left./ \mathrm{m}^{2}\right)$.

$\mathrm{F}_{s 2}$ - radiação solar direta complementar ascendente (Watt/ $/ \mathrm{m}^{2}$ ).

$\mathrm{F}_{\text {sotu }}$ - radiação do solo (Watt/m²).

G - fluxo de calor no solo (Watt/ $\left.\mathrm{m}^{2}\right)$.

$h \rightarrow$ altura da vegetação $(m)$.

I $\rightarrow$ intensidade de radiação (Watt/ $\left.\left(\mathrm{m}^{2} \mathrm{sr}\right)\right)$.

$\mathrm{K}_{\mathrm{m}} \rightarrow$ difusividade turbulenta para a quantidade de movimento $\left(\mathrm{m}^{2} / \mathrm{s}\right)$.

$\mathrm{K}$ - difusividade turbulenta de calor sensível $\left(\mathrm{m}^{2} / \mathrm{s}\right)$.

$\mathrm{K}_{c}$ - difusividade turbulenta do vapor d’águat $\left(\mathrm{m}^{2} / \mathrm{s}\right)$.

L $\rightarrow$ comprimento de estabilidade de Monin-Obukhov ( $m$ ).

$L_{v} \rightarrow$ ealor latente de vaporização (Joule/kg).

$\mathrm{M}_{\mathrm{v}} \rightarrow$ peso molecular do vapor d'água (0.018 kg/mole).

$p(z)$ - solução particular da equaçĩo diferencial de Ricatti.

$\mathrm{q} \rightarrow$ umidade do ar $(\mathrm{g} / \mathrm{kg})$.

$\mathrm{R}^{*}$ - constante dos gases (8.31 Joule/('K.mol).

$\mathrm{R}(\mathrm{z})$ - resistência aerodinâmica (s/ $\mathrm{m})$.

$\mathrm{r}_{\mathrm{b}}$ - resistência de camada limite ( $\mathrm{s}$ ) $\mathrm{m})$.

$r_{s} \rightarrow$ resistência estomática $(\mathrm{s} / \mathrm{m})$

$\mathrm{S}^{*} \rightarrow$ fontes locais de calor sensível (Watt $/ \mathrm{m}^{3}$ ).

$\mathrm{T}(\mathrm{z}) \rightarrow$ temperatura do ar ('K).

$\mathrm{T}_{\mathrm{v}} \rightarrow$ temperatura das superfícies vegetais ("K).

$t$ - tempo (s).

u $\rightarrow$ velocidade do vento $(\mathrm{m} / \mathrm{s})$.

$\mathrm{u}_{*} \rightarrow$ velocidade de fricção $(\mathrm{m} / \mathrm{s})$.

$\mathrm{V}$ - fontes locais de vapor d'água (Watt $\left./ \mathrm{m}^{\mathrm{i}}\right)$.

$\mathrm{z}$ - coordenada vertical (m).

$\alpha$ - coeficiente de atenuação da radiaçã̃o do solo.

$\gamma \rightarrow$ constante psicrométrica.

$\Delta-$ de / dT .

$\varepsilon-$ coeficiente de emissão de radiação de onda longa das superfícies vegetais.

$\theta \rightarrow$ temperatura potencial do ar ("K).

$\kappa$ - constante de von Karman (0.4).

$v \rightarrow$ viscosidade cinemática $\left(\mathrm{m}^{2} / \mathrm{s}\right)$.

$\rho \rightarrow$ densidade do ar $\left(\mathrm{kg} / \mathrm{m}^{3}\right)$.

$\sigma \rightarrow$ constante de Stefan-Boltzmann (Watt/(m- $\left.\mathrm{m}^{2} \mathrm{~K}^{4}\right)$ ). 


\section{ANEXO}

Composição das matrizes (equações 8,11 e 12)

Vetores [S] ou [V]

Para $\mathrm{i}=0, \ldots \mathrm{n}$

Matrizes $\left[\mathbf{R}+\mathbf{r}_{\mathrm{c}}\right]$ ou $\left[\mathbf{R}+\mathbf{r}_{\mathrm{d}}\right]$

Para $\mathrm{i}<\mathrm{j}$ ou $\mathrm{i}=\mathrm{j}=0 \rightarrow$

Para $i>j$

Para $\mathrm{i}=\mathrm{j}>0$

\section{Matriz [R]}

Para ij e $\mathrm{j}>0$

Para $j=0$

Para $\mathrm{i}>\mathrm{j}$ e $\mathrm{j}>0$

Matrizes $\left[\mathbf{r}_{\mathrm{c}}\right]$ ou $\left[\mathbf{r}_{\mathrm{d}}\right]$

Para $\mathrm{i}=\mathrm{j}$ ou $\mathrm{i}=\mathrm{j}=0$

Para $\mathrm{i}=\mathrm{j}>0$

\section{Vetor $\left[\Delta \mathbf{F}_{1}\right]$}

Para $\mathrm{i}=0$

Para $\mathrm{i}=1, \ldots \mathrm{n}$

\section{Matriz [AB]}

Para $\mathrm{i}=\mathrm{j}=0$

Para $\mathrm{i}>0$ e $\mathrm{j}=0$

Para $\mathrm{i}=0 \mathrm{e} \mathrm{j} 0$

Para $\mathrm{i}>0 \mathrm{e} \mathrm{i}<\mathrm{j}$

Para $\mathrm{i}>\mathrm{j}$ e $\mathrm{j}>0$

Para $\mathrm{i}=\mathrm{j}>0$

Matriz $\left[a_{i}, z\right]$

Para $\mathrm{i}=\mathrm{j}=0$

Para $\mathrm{i}=\mathrm{j}>0$

Para $\mathrm{i}=\mathrm{j}$

$$
(n+1) \cdot 1
$$

$\rightarrow \quad \mathrm{S}_{1}$ ou $\mathrm{V}_{\mathrm{i}}$

$$
n+1) \cdot(n+1)
$$

$\mathrm{R}_{\mathrm{j}}$

$\rightarrow \quad 2 R_{i}-R_{i}$

$\rightarrow \quad\left(\mathrm{R}_{\mathrm{i}}+2 \cdot \mathrm{r}_{\mathrm{cl}}\right)$ ou $\left(\mathrm{R}_{\mathrm{i}}+2 \cdot \mathrm{r}_{\mathrm{di}}\right)$

$(n+1) \cdot(n+1)$

$\begin{array}{ll}- & \mathrm{R} \\ - & 0\end{array}$

$\rightarrow \quad 2 R_{1}-R_{1}$

$(n+1) \cdot(n+1)$

0

$r_{\mathrm{si}}$ ou $r_{\mathrm{di}}$

$(n+1) \cdot 1$

$\rightarrow \quad \mathrm{F}_{(10) \mid}$

$\rightarrow \quad \Delta \mathrm{F}_{\mathrm{l}(i)}$

$(n+1) \cdot(n+1)$

$-\quad-1$

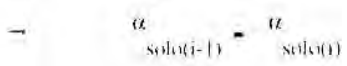

$\rightarrow \quad \mathrm{A}_{0,1}$

$-A_{i, j}-A_{i-1.1}$

- $\mathrm{B}_{1+1.1}-\mathrm{B}_{\mathrm{i}, \mathrm{A}}$

$-\left(B_{1.1}+A_{1.1 .1 .1}\right)$

$$
(n=1) \cdot(n+1)
$$

1

a. $\Delta z$

0 